\title{
Experimental observation of effect of the wall curvature of capillary tube on colloidal crystallization inside the tube
}

\author{
Shenwei Wang ${ }^{\mathrm{a}, \mathrm{b}}$, Hongwei Zhou ${ }^{\mathrm{a}}$, Xiaoan Zhao ${ }^{\mathrm{a}, \mathrm{b}}$, Shenghua $\mathrm{Xu}^{\mathrm{a}, \mathrm{b}, *}$ \\ ${ }^{a}$ Key Laboratory of Microgravity (National Microgravity Laboratory), Institute of Mechanics, Chinese Academy of Sciences, Beijing, 100190, China \\ ${ }^{\mathrm{b}}$ School of Engineering Science, University of Chinese Academy of Sciences, Beijing, 100049, China
}

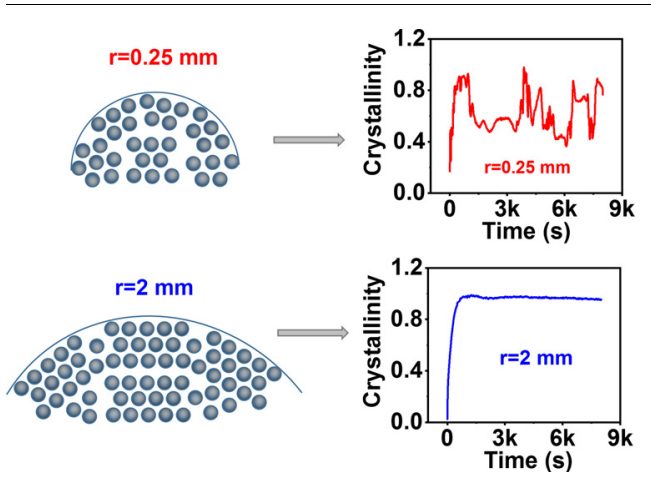

A R T I C L E I N F O

\section{Keywords:}

Colloidal suspension

Crystallization process

Capillary

Wall curvature

\begin{abstract}
A B S T R A C T
We investigated the crystallization process of colloidal suspensions in capillary tubes with different curvature radius. The evolution of structural parameters during crystallization such as crystallinity, average crystallites size and number of crystallites were determined by the reflection spectrum combined with a specially developed data-handling method. We found that the wall curvature has a significant effect on fluctuation, thus the stability of the structural parameters during crystallization process, and with the decrease of the curvature, this effect quickly weakens. In addition, our experiments showed the concentration has little influence on crystal stability.
\end{abstract}

\section{Introduction}

Homogeneous crystallization has been studied more widely than heterogeneous crystallization although, in many cases, the latter not only is inevitable to take place but also often plays a more dominant role [1-5]. In fact, one can also use heterogeneous crystallization to produce desired products. Up to now, how to understand and control the heterogeneous crystallization process is still a challenging task. In this respect, colloidal crystals have wide potential applications such as photonic band gap materials [6-8], optical filters [9-11], sensors [12-14] and also colloids are an excellent model system for atoms [15-17], thus to get information from colloidal heterogeneous crystallization has attracted one's attention. In general, heterogeneous nucleation can be realized by seeds or container walls [18-22]. Among the various factors affecting heterogeneous crystallization, the curvature of walls may induce geometrical frustration and non-uniform stress in crystals, hence the final crystals formed on curved walls may exhibit unusual crystallization process and configuration [23,24].

* Corresponding author at: Key Laboratory of Microgravity (National Microgravity Laboratory), Institute of Mechanics, Chinese Academy of Sciences, Beijing, 100190, China.

E-mail address: xush@imech.ac.cn (S. Xu). 
Sandomirski et al. [25] investigated the heterogeneous crystallization of colloidal spheres near cylindrical and spherical seeds from particle-level information obtained by confocal microscopy and computer simulations, with cylinder radius up to 546 times particle radius and sphere radius up to 1370 times particle radius. The results showed that the crystallites growth rate increased with the decrease of curvature, and curved walls of cylindrical and spherical seeds led to distortions and accumulating elastic stress as the crystallites grow. Computer simulations also showed that crystal growth on curved space has elastic instabilities driven by curvature induced stress [26], and crystals grown preferentially in regions of low curvature and the lattice frustration produced in regions of high curvature for a wall with sinusoidal surface [27]. In order to further understand the influence of curved walls on the crystallization kinetics, it would be desirable to obtain the information about how the relevant structural parameters evolve with time during crystal growth.

In this study, we investigated the crystallization process in the suspension of charged colloidal particles in tubes with different curvature radius. The evolution of structural parameters including crystallinity, average crystallites size and number of crystallites, etc. during crystallization were monitored by a reflection spectrum combined with a specially developed data-handling method in a non-invasive, realtime and in-situ manner. Our observations showed that fluctuations of these structural parameters increase with curvature during crystallization but little dependence with concentration of suspensions.

\section{Materials and methods}

\subsection{Materials}

The negatively charged polystyrene particles with sulfonic groups on their surface were purchased from Huge Biotechnology (Shanghai, China). The received particles were purified and filtrated again by repeated washing with ultrapure water (UPRLC5DRO UPW system, Relatec, China) in the centrifugation process, and then stored with ionexchange resin (Amberlite IRN-150, EMD Millipore Corporation, Germany) for further deionization. The mean diameter and polydispersity of the particles determined by dynamic light scattering (Brookhaven Instruments Corporation, USA) are $85 \mathrm{~nm}$ and $4 \%$, respectively. The sample suspensions (volume fraction: $\Phi=0.5 \%, 1 \%$ and $2 \%$ ) were prepared by carefully mixing the as-prepared suspension with a certain amount of ultrapure water. The densities of water and polystyrene colloidal particles are $1 \mathrm{~g} / \mathrm{cm}^{3}$ and $1.05 \mathrm{~g} / \mathrm{cm}^{3}$, respectively. The roughly matched density and small size of $85 \mathrm{~nm}$ particles ensure that the precipitation effect can be ignored. Experiments were made at $25 \pm 2{ }^{\circ} \mathrm{C}$ in an air-conditional room.

\subsection{Experiments}

The experimental setup used, which was the same that described in our previous paper [28,29], consists of a reflection spectrometer, a crystallization cell, a peristaltic pump and an ion-exchange chamber as shown in Fig. 1. A light beam from a tungsten halogen light source (Avalight-HAL, Avantes, Netherlands) hits the cell wall through a Ytype optical fiber cable and the reflection spectra at the angle of $180^{\circ}$ are taken on a optical spectrometer (Avaspec-2048, Avantes, Netherlands). The wavelength range of the spectral measurement is $400 \mathrm{~nm}-1100 \mathrm{~nm}$. The beam size for the reflection spectrum is about $1 \mathrm{~mm}$ and the penetration depth of the focus beam is basically near the wall unless special measures are taken. To check whether there is any temperature change of sample due to possible heating effect of the light beam, we placed a temperature detector at the optical fiber head and found that the variation range of its temperature around the average value $<0.5^{\circ} \mathrm{C}$ within two hours, thus confirming that the heating effect on the sample can be ignored. The peristaltic pump can circulate colloidal suspension through the ion-exchange chamber to deionize the suspension continuously. To investigate the effect of curved walls on crystallization, the crystallization cell consists of four capillary tubes with different curvature radius $(r=0.25 \mathrm{~mm}, 0.5 \mathrm{~mm}, 1 \mathrm{~mm}, 2 \mathrm{~mm})$ and an additional square tube with $1 \mathrm{~mm}$ side width. When the colloidal suspension is passed into the narrow crystallization cell, the crystals are melted away by applying a shear flow. After stopping the flow, the crystallization process can be recorded by tracing the reflection intensity changes.

\section{Results and discussion}

The structural parameters during crystal growth are analyzed from the measured reflection spectra at different time. A typical change of the reflection spectra for colloidal suspension with $\Phi=0.5 \%$ in a tube is shown in Fig. 2. The reflection peak intensity increased rapidly with time in the crystallization process, indicating the increase of crystal size. Based on the spectra, we can determine the crystal structures from the wavelengths of reflection peaks originating from different crystal planes $[29,30]$. Since the wave vectors $\left(q=\left(\frac{4 \pi n}{\lambda}\right) \sin \left(\frac{\theta}{2}\right), \theta=180^{\circ}\right)$ corresponding to these peaks are $16.23,28.09,32.45 \mu \mathrm{m}^{-1}$, and the ratio is $\sqrt{2} / \sqrt{6} / \sqrt{8}$, the corresponding crystal planes are (110) plane, (211) plane, (220) plane and the crystal structure is body centered cubic (bcc). From analyzing reflection spectra at different time, it can be concluded that the crystal structure maintained unchanged in the crystallization process. The primary peak wavelength $\lambda$, which is related to the value of the nearest-neighbor interparticle distance $D$ based on Eq. (1) where $n$ is the refractive index, decreased at the beginning of crystallization and then maintained constant. The shift of the primary peak wavelength indicates that the crystals become more stable and compact during crystallization.

$D=0.6124 \lambda / n$

To further quantitatively understand the crystallization process of colloidal suspension on curved walls, some important parameters such as crystallinity $X(t)$, average crystallites size $L(t)$ and number of crystallites $N_{c}(t)$ are used to study the crystallization kinetics, which can be evaluated as follows [31]:

(2) The crystallinity $X(t)$ represents the fraction of the sample which has become crystalline from liquid, it can be used as an indicator to show the degree of crystallization. $X(t)=0$ means the sample is in liquid state and there is no crystalline state, and $X(t)=1$ means all the liquid part has been transformed into crystalline state. $X(t)$ is calculated from the reflection peak area $A(t)$ by

$X(t)=c A(t)$

where $\mathrm{c}$ is the normalization factor, which is based on the maximum value of the primary peak area during crystallization.

(3) The average crystallites size $L(t)$ can be calculated from the full width at half maximum of the primary peak (FWHM) by

$L(t)=2 \pi K / \Delta q(t)$

where $K=1.15$ is the Scherrer constant for crystallites, $\Delta \mathrm{q}(\mathrm{t})$ is the full width at half maximum primary peak.

(4) The number of crystallites $N_{c}(t)$ is estimated by

$N_{c}(t)=X(t) / L^{3}(t)$

The influences of the curved walls and particle concentration on the crystallization process in the tube are systematically studied in the Sections 3.1 and 3.2.

\subsection{Crystallization process on the curved walls}

For the same colloidal suspension with $\Phi=0.5 \%$, four different curvature tubes $(r=0.25 \mathrm{~mm}, 0.5 \mathrm{~mm}, 1 \mathrm{~mm}, 2 \mathrm{~mm}$ ) were used for investigating the influence of tube curvature on crystallization. The 


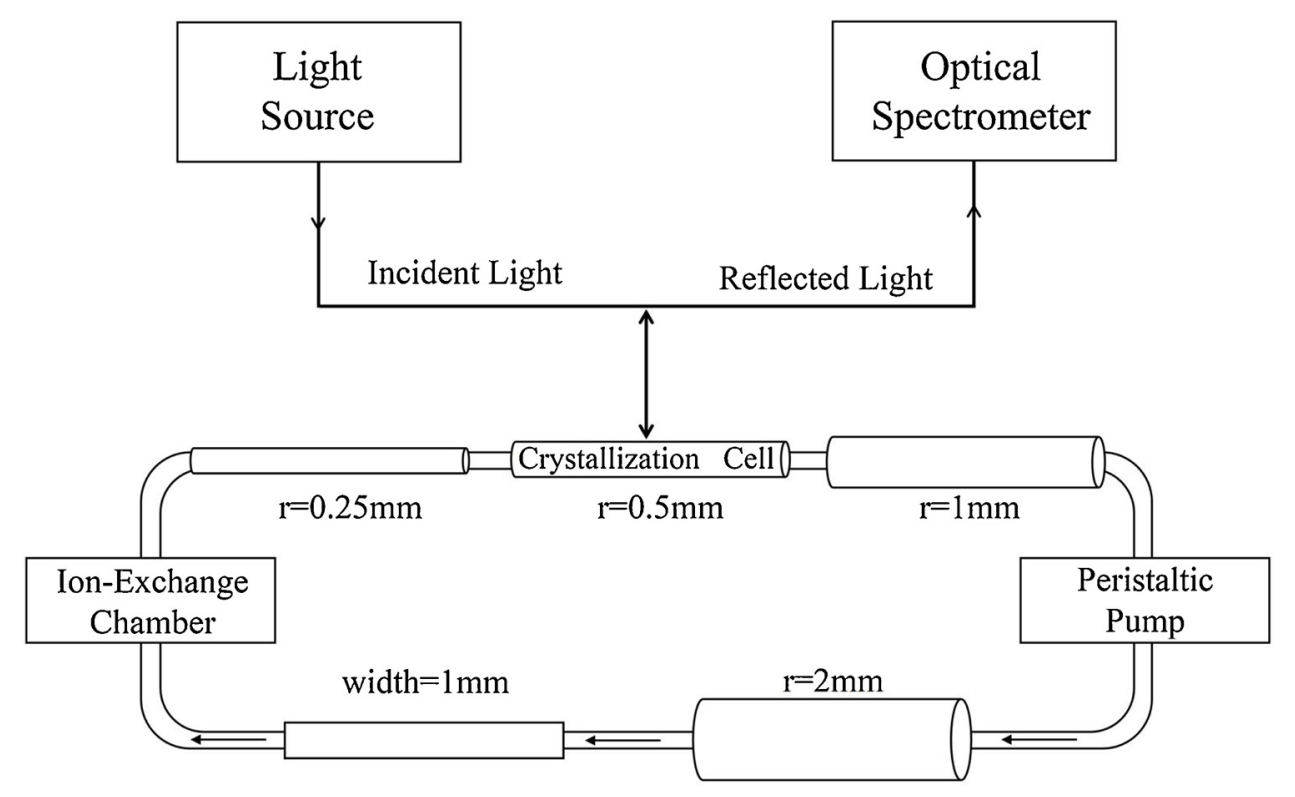

Fig. 1. Schematic representation of the measuring apparatus.
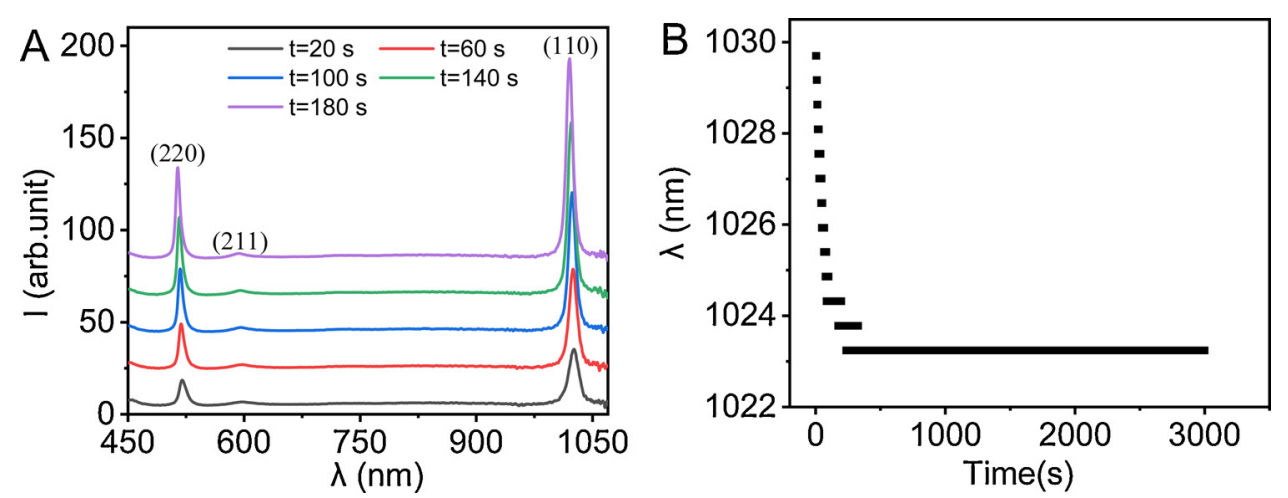

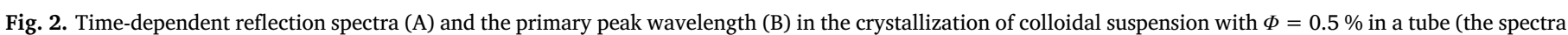
were shifted for clarity).

variations of structural parameters $X(t), L(t)$ and $N_{c}(t)$ in these tubes are shown in Fig. 3. From these trends we can see that there are large differences in crystallization kinetics in the tube with different curvatures, which are described as follows:

(I) The crystallinity $X(t)$ represents the fraction of the sample which has become crystalline from liquid. For the tube of $r=0.25 \mathrm{~mm}, X$ $(t)$ increased from 0 to 0.9 at the beginning of crystallization, which means the samples almost become crystals from liquid. And then $X(t)$ decreased and showed great fluctuations between 0.4 and 1 , indicating that the crystals are very unstable in the tube of $r=0.25 \mathrm{~mm}$ and the transformation between the ordered phase and the disordered phase kept taking place. For the tubes of $r=0.5 \mathrm{~mm}$ and $r=1 \mathrm{~mm}$, the crystallinity also showed the similar changing tendency, that is, $X(t)$ increased first and then showed fluctuation. But the degree of fluctuation for different curvature tubes is different. The results of Fig. 3 showed that the larger the radius of the tube, the less the degree of fluctuation. For the tube of $r=2 \mathrm{~mm}, X(t)$ increased from 0 to 1 and there is no fluctuation, indicating that the crystals are stable in the tube of $r=2 \mathrm{~mm}$, similar to that in the square tube (namely, an infinite radius of curvature). In order to quantitatively describe the degree of fluctuation of structural parameters, we calculated the coefficient of variation of the structural parameters, which is defined as the ratio of the standard deviation to the mean. The mean, standard deviation and coefficient of variation of structural parameters were presented in Table 1 . For four different curvature tubes of $r=0.25 \mathrm{~mm}, 0.5 \mathrm{~mm}, 1 \mathrm{~mm}$ and $2 \mathrm{~mm}$, the coefficients of variation of the crystallinity are $0.22,0.08,0.04,0.008$, further indicating that the degree of fluctuation showed considerable variation in different curved tubes.

(II) The average crystallites size $L(t)$ grew dramatically from the beginning of crystallization and then maintained fluctuation when tube radius is relatively small, but the degree of the average crystallites size fluctuation is less than that of crystallinity fluctuation. Besides, the average crystallites size increased with increasing the tube radius. The average crystallites size were $24 \mu \mathrm{m}$, $26 \mu \mathrm{m}, 32 \mu \mathrm{m}$ and $39 \mu \mathrm{m}$ in the tubes of $r=0.25 \mathrm{~mm}, 0.5 \mathrm{~mm}$, $1 \mathrm{~mm}$ and $2 \mathrm{~mm}$. The coefficients of variation of the crystallites size were $0.06,0.03,0.028$ and 0.03 in the tubes of $r=0.25 \mathrm{~mm}$, $0.5 \mathrm{~mm}, 1 \mathrm{~mm}$ and $2 \mathrm{~mm}$, respectively.

(III) The number of crystallites $N_{c}(t)$ also showed different degrees of fluctuation in the four different tubes and roughly decreased with increasing the tube radius, which were $4.32 * 10^{13} \mathrm{~m}^{-3}$, $4.7 * 10^{13} \mathrm{~m}^{-3}, 2.6^{*} 10^{13} \mathrm{~m}^{-3}$ and $1.6 * 10^{13} \mathrm{~m}^{-3}$ in the tubes of $r=0.25 \mathrm{~mm}, 0.5 \mathrm{~mm}, 1 \mathrm{~mm}$ and $2 \mathrm{~mm}$. The coefficients of variation were $0.19,0.11,0.12$ and 0.1 in the tubes of $r=0.25 \mathrm{~mm}$, $0.5 \mathrm{~mm}, 1 \mathrm{~mm}$ and $2 \mathrm{~mm}$, respectively.

The structure fluctuation of colloidal crystals in four curved tubes 

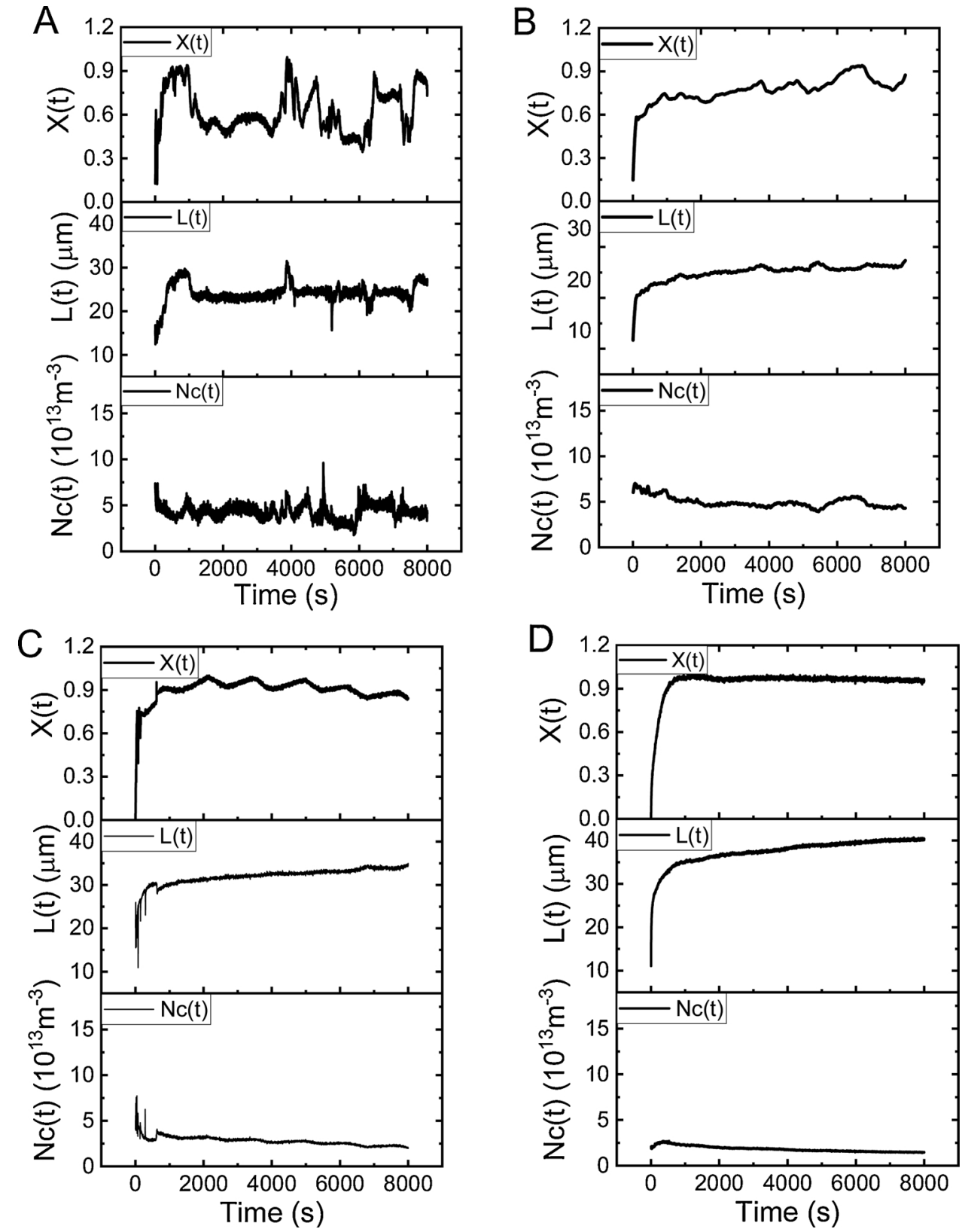

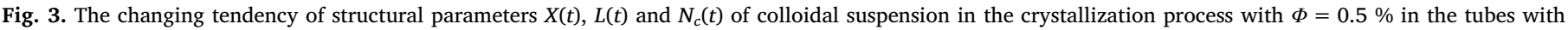
different curvature ( $r=0.25 \mathrm{~mm}, 0.5 \mathrm{~mm}, 1 \mathrm{~mm}, 2 \mathrm{~mm}$ for A-D, respectively).

Table 1

The mean, standard deviation and coefficient of variation of structural parameters (crystallinity, crystallites size and crystallites number).

\begin{tabular}{lllll}
\hline$r(\mathrm{~mm})$ & 0.25 & 0.5 & 1 & 2 \\
\hline Mean of crystallinity & 0.611 & 0.8 & 0.91 & 0.96 \\
SD of crystallinity & 0.137 & 0.063 & 0.038 & 0.008 \\
CV of crystallinity & 0.22 & 0.08 & 0.04 & 0.008 \\
Mean of crystallites size $(\mu \mathrm{m})$ & 24 & 26 & 32 & 39 \\
SD of crystallites size $(\mu \mathrm{m})$ & 1.53 & 0.78 & 0.89 & 1.16 \\
CV of crystallites size & 0.06 & 0.03 & 0.028 & 0.03 \\
Mean of crystallites number $\left(* 10^{13} \mathrm{~m}^{-3}\right)$ & 4.32 & 4.7 & 2.6 & 1.6 \\
SD of crystallites number $\left(* 10^{13} \mathrm{~m}^{-3}\right)$ & 0.83 & 0.48 & 0.31 & 0.16 \\
CV of crystallites number & 0.19 & 0.11 & 0.12 & 0.1 \\
\hline
\end{tabular}

SD: Standard Deviation; CV: Coefficient of Variation.

showed great differences. To understand such structure fluctuations with tube curvatures, as a comparison, we also examined the crystallization process in a square tube with a width of $1 \mathrm{~mm}$. As shown in Fig. 4, the structure parameters $X(t), L(t)$ and $N_{c}(t)$ of colloidal suspension in the crystallization process in the square tube presented different fluctuation behavior with that in tube of $r=0.5 \mathrm{~mm}$. We can see that the $X(t), L(t)$ and $N_{c}(t)$ all maintained constant in the late stage of crystallization, indicating that the crystals in the square tube (flat surface) is stable, and therefore confirm that the structure fluctuation in the tube is associated with the curvature of the tube.

In presence of a wall during nucleation, the most closely packed crystal plane (e.g., (111) plane for fcc and (110) plane for bcc) will tend to be parallel to the wall [32]. In curved tube, crystal nucleus are more likely to form on the smooth curved wall due to the lower nucleation free energy barrier [21]. Although the wall favor crystallization, the curvature of the tube leads to curved crystal planes and hence results in a mismatch between the thermodynamically favored and curved wallinduced crystal structure, which leads to elastic distortions in the growing crystallite [24-26]. 


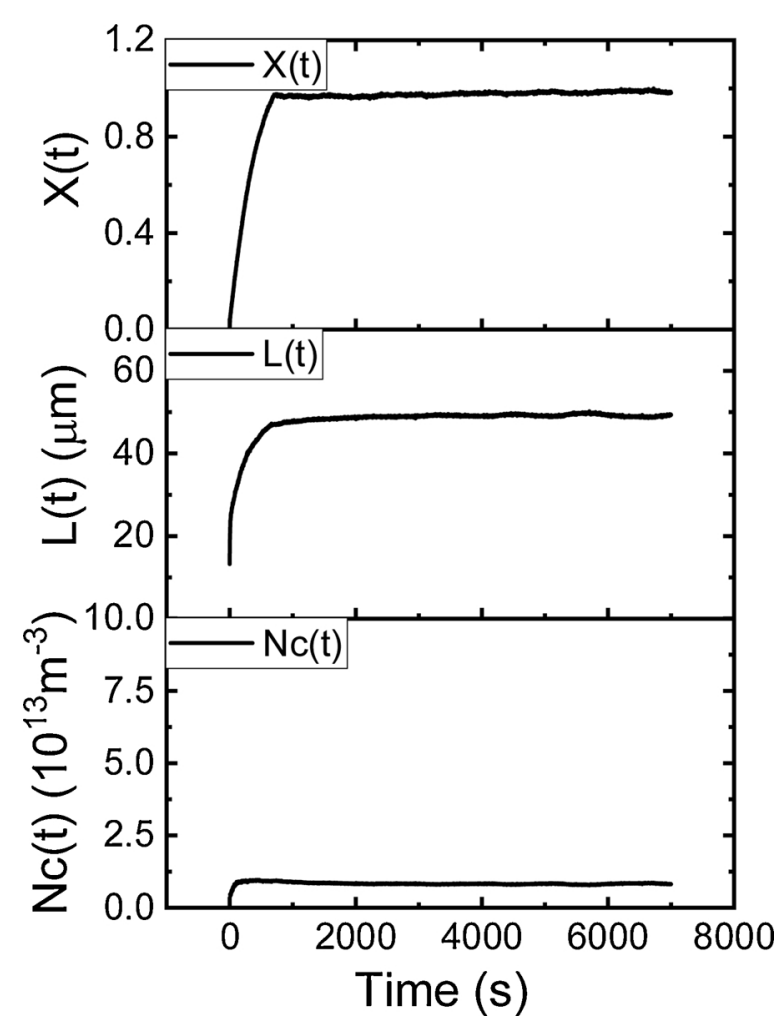

Fig. 4. The changing tendency of structural parameters $X(t), L(t)$ and $N_{c}(t)$ of colloidal suspension in the crystallization process with $\Phi=0.5 \%$ in a square tube with a width of $1 \mathrm{~mm}$.

The stability of wall-induced crystallite is mainly determined by the competition between the elastic energy penalty and the interfacial energy [33]. The crystallite detached from the curved wall once the elastic energy penalty reaches the interfacial energy. On the contrary, the crystallite can maintain stable state if the elastic energy penalty does not reach the interfacial energy. For colloidal system, elasticity theory predicts for the elastic stress [33,34]

$\mathrm{F}_{e}=\frac{E}{96} \frac{L^{5}}{(r+L)^{2}}$

where $E$ is the bulk modulus of the crystallite, $L$ is the crystallite size and $r$ is the wall curvature radius. The interfacial energy is presented as:

$\mathrm{F}_{i}=\frac{\pi L^{2}}{4} \Delta \gamma$

where $\Delta \gamma$ is the difference in interfacial tensions. For the case of flat walls where $r \rightarrow \infty, F_{e}=0$ so that there is no curved wall-induced distortions, and crystallite of any size can remain stable. For smaller wall curvature radius $r$, the stable crystallite $L$ should also be smaller to ensure that the elastic energy penalty does not reach the interfacial energy, according to Eqs. (5) and (6). Therefore, the crystallite is easier to detach from the wall and the crystallite size is smaller for smaller $r$. When the stress induced by curvature was released through detachment, the crystallite can grow again on the curved wall due to the lower nucleation free energy barrier, so that the growth and detachment of the crystallite on the curved wall occur alternately and continuously, resulting in fluctuations in $X(t)$ and other parameters. Such a model can qualitatively describe the mechanics of fluctuations in this study.

\subsection{Crystallization process with different concentration on the curved walls}

The crystallization process of colloidal suspension showed great differences in curved tubes with different curvature, especially for the tubes of $r=2 \mathrm{~mm}$ and $r=0.25 \mathrm{~mm}$. In this section, we investigated the effect of concentration on the crystallization of colloidal suspension in the tubes of $r=2 \mathrm{~mm}$ and $r=0.25 \mathrm{~mm}$. The crystal structures for concentration of $\Phi=0.5 \%, 1 \%$ and $2 \%$ are all bcc.

For the tube of $r=2 \mathrm{~mm}$, the changing tendency of structural parameters $X(t), L(t)$ and $N c(t)$ in the crystallization process with different concentration ( $\Phi=0.5 \%, 1 \%$ and $2 \%$ ) are showed in Fig. 5 . We have discussed the variations of structural parameters $X(t), L(t)$ and $N_{c}$ ( $t$ ) for the concentration of $\Phi=0.5 \%$ in the 3.1 section. By Comparing the crystallization process of three kinds of concentration $(\Phi=0.5 \%, 1$ $\%$ and $2 \%$ ), we found that structural parameters $X(t), L(t)$ and $N c(t)$ all showed similar variation tendency for suspensions with three different concentrations. The crystallinity $X(t)$ increased from 0 to 1 and maintained almost unchanged with no fluctuation, which means the crystallites have stable state. The average crystallites size $L(t)$, which increased first and maintained almost constant, were $39 \mu \mathrm{m}, 21 \mu \mathrm{m}$ and $9 \mu \mathrm{m}$ for suspensions $\Phi=0.5 \%, 1 \%$ and $2 \%$. The number of crystallites $N_{c}(t)$, which maintained almost constant, were $1.6^{*} 10^{13} \mathrm{~m}^{-3}$, $10.4 * 10^{13} \mathrm{~m}^{-3}$ and $133 * 10^{13} \mathrm{~m}^{-3}$ for suspensions $\Phi=0.5 \%, 1 \%$ and $2 \%$.

Similarly, for the tube of $r=0.25 \mathrm{~mm}$, the variations of structural parameters $X(t), L(t)$ and $N c(t)$ in the crystallization process with different concentration $(\Phi=0.5 \%, 1 \%$ and $2 \%)$ are presented in Fig. 6 . From these trends we can see that the whole crystallization process also seems to have similar changing tendency. Both the crystallinity $X(t)$ and the average crystallites size $L(t)$ increased firstly at the beginning of crystallization and then fluctuated largely, the number of crystallites $N_{c}$ $(t)$ also shown fluctuation in the crystallization process. As the concentration increases, the average crystallites size $L(t)$ decreased and the number of crystallites $N_{c}(t)$ increased.

The structural parameters during crystallization process showed similarity, especially for fluctuation behavior, for different concentration in the tubes of $r=2 \mathrm{~mm}$ and $0.25 \mathrm{~mm}$, indicating that the
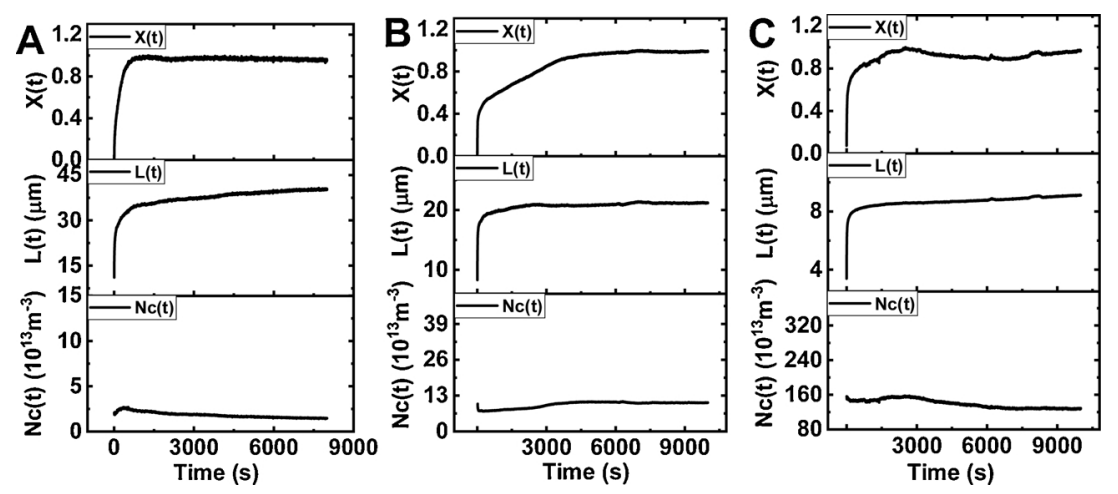

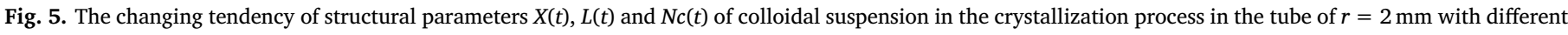
concentration ( $\Phi=0.5 \%, 1 \%$ and $2 \%$ for A-C, respectively). 

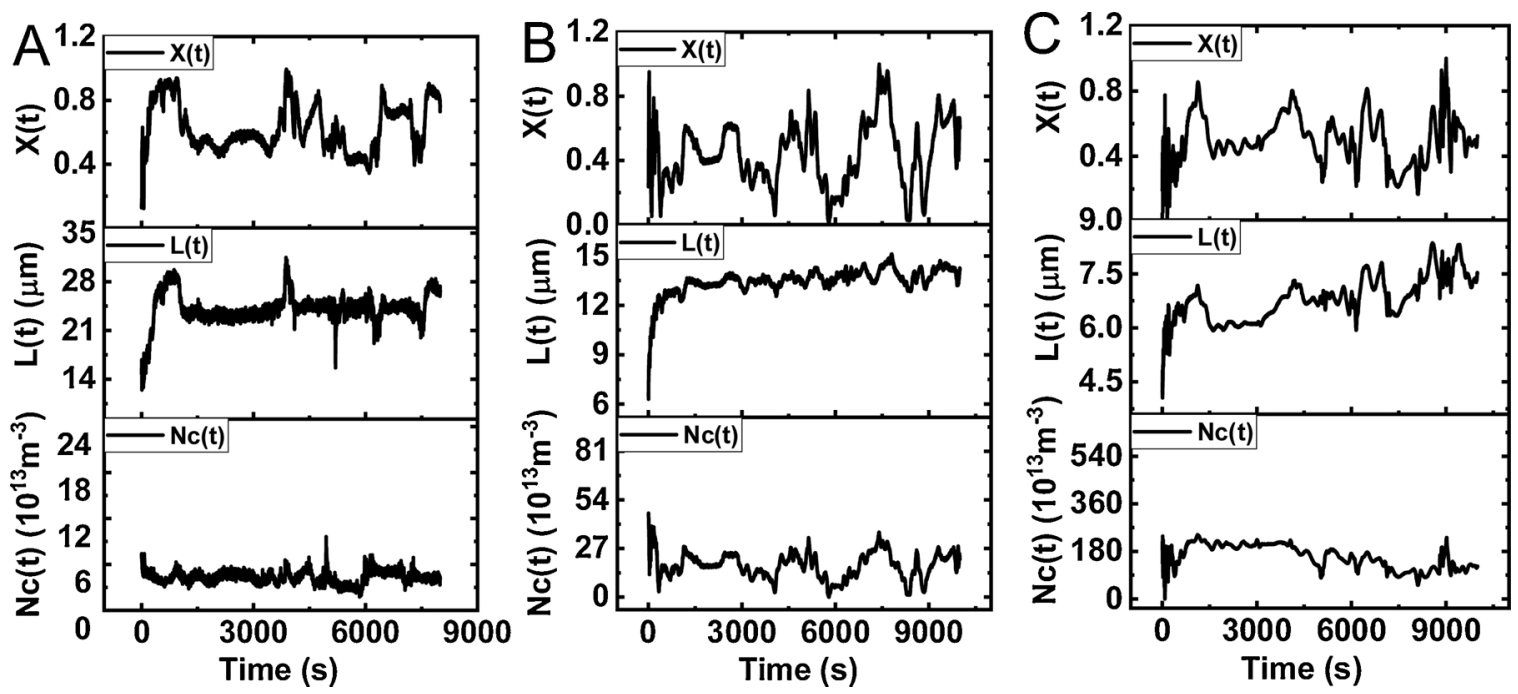

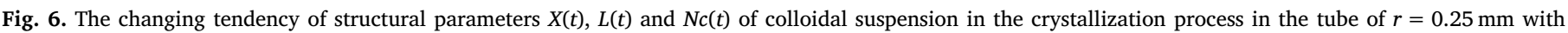
different concentration ( $\Phi=0.5 \%, 1 \%$ and $2 \%$ for A-C, respectively).

concentration has almost no effect on the stability of the crystallites. The fluctuation behavior of the structural parameters is due to the change in crystal state, which resulted from the accumulated elastic stress in curved crystal planes. With the increase of concentration, a combination of increasing crystallites number and decreasing crystallites size leads to little effect of concentration on crystal state.

\section{Conclusion}

In this study, by means of the reflection spectrum combined with a specially developed data-handling method, we carried out real-time insitu experimental observation on the evolution process of a set of parameters characterizing the crystallization in capillary tubes with different curvature radius. These parameters include the crystallinity, the average crystallites size and the number of the crystallites. We found that the tube curvature has effect on the fluctuation amplitude, thus the stability of the structural parameters during crystallization process, directing the effect rooted in the crystallization kinetics. The larger the curvature, the more obvious the effect is. When the curvature radius of the tube reaches $2 \mathrm{~mm}$ or larger, this effect becomes almost negligible. The structural instability caused by surface curvature can qualitatively be explained by the competition between the elastic energy penalty and the interfacial energy. It is also found that the increase of concentration has little effect on the stability of the crystallites except making the decrease of the average crystallites size and the increase of the number of crystallites.

The assembly of colloidal building-blocks is an ascendant approach in recent years for the fabrication of a wide variety of functional materials, such as photonic crystals, with designed shapes and large areas. We hope that our work could provide reference: when designing and preparing colloidal crystal materials, attention should be paid to the problem that large curvature surface may cause structural defects.

\section{CRediT authorship contribution statement}

Shenwei Wang: Investigation, Writing - original draft, Writing review \& editing. Hongwei Zhou: Validation, Formal analysis. Xiaoan Zhao: Investigation. Shenghua Xu: Conceptualization, Supervision, Funding acquisition.

\section{Declaration of Competing Interest}

The authors declare that they have no known competing financial interests or personal relationships that could have appeared to influence the work reported in this paper.

\section{Acknowledgements}

This work was supported by the National Natural Science Foundation of China (Grant Nos. 11672295 and U1738108), and Key Research Program of Frontier Sciences, CAS, Grant NO. QYZDY-SSWJSC040.

\section{References}

[1] J. Cai, J.C. E, M.X. Tang, X.R. Zhu, Y. Cai, S.N. Luo, Crystallization of Lennard-Jones liquids under dynamic compression: heterogeneous and homogeneous nucleation, J. Chem. Phys. 147 (2017) 7.

[2] J.R. Espinosa, C. Vega, C. Valeriani, D. Frenkel, E. Sanz, Heterogeneous versus homogeneous crystal nucleation of hard spheres, Soft Matter 15 (2019) 9625-9631.

[3] K. Harano, T. Homma, Y. Niimi, M. Koshino, K. Suenaga, L. Leibler, E. Nakamura, Heterogeneous nucleation of organic crystals mediated by single-molecule templates, Nat. Mater. 11 (2012) 877-881.

[4] M.P. Howard, S.T. Milner, A simple model for heterogeneous nucleation of isotactic polypropylene, Macromolecules 46 (2013) 6593-6599.

[5] W.S. Xu, Z.Y. Sun, L.J. An, Heterogeneous crystallization of hard spheres on patterned substrates, J. Chem. Phys. 132 (2010) 7.

[6] A. Chaouachi, R. Chtourou, A. M'Nif, A.H. Hamzaoui, Optical characterization of colloidal silica crystals with controlled size microspheres, Mater. Lett. 116 (2014) 420-424.

[7] C. Chen, Z.Q. Dong, Y. Xu, X.H. Wang, H. Lu, Y.F. Qiu, Z.G. Zhu, Ultrathin colloidal crystal layer as transparent photonic films, Micro Nano Lett. 14 (2019) 1-4.

[8] M. Stimulak, M. Ravnik, Tunable photonic crystals with partial bandgaps from blue phase colloidal crystals and dielectric-doped blue phases, Soft Matter 10 (2014) 6339-6346.

[9] C. Chen, Z.-Q. Dong, Y. Xu, X.-H. Wang, H. Lu, Y.-F. Qiu, Z.-G. Zhu, Ultrathin colloidal crystal layer as transparent photonic films, Micro Nano Lett. 14 (2019) 1-4.

[10] H.S. Lee, T.S. Shim, H. Hwang, S.-M. Yang, S.-H. Kim, Colloidal photonic crystals toward structural color palettes for security materials, Chem. Mater. 25 (2013) 2684-2690.

[11] Y. Sugao, S. Onda, A. Toyotama, Y. Takiguchi, T. Sawada, S. Hara, S. Nishikawa, J. Yamanaka, Gelled colloidal crystals as tunable optical filters for spectrophotometers, Jpn. J. Appl. Phys. 55 (2016).

[12] S. Jia, Z. Tang, Y. Guan, Y. Zhang, Order-disorder transition in doped microgel colloidal crystals and its application for optical sensing, ACS Appl. Mater. Interfaces 10 (2018) 14254-14258.

[13] W. Tang, C. Chen, Hydrogel-based colloidal photonic crystal devices for glucose sensing, Polymers 12 (2020).

[14] F. Wang, Z. Zhu, M. Xue, F. Xue, Q. Wang, Z. Meng, W. Lu, W. Chen, F. Qi, Z. Yan, Cellulose photonic crystal film sensor for alcohols, Sens. Actuators B-Chem. 220 (2015) 222-226.

[15] D.M. Herlach, Colloids as model systems for metals and alloys: a case study of crystallization, Eur. Phys. J.-Spec. Top. 223 (2014) 591-608.

[16] B. Li, D. Zhou, Y. Han, Assembly and phase transitions of colloidal crystals, Nat. Rev. Mater. 1 (2016). 
[17] D. Taha, S.R. Dlamini, S.K. Mkhonta, K.R. Elder, Z.-F. Huang, Phase ordering, transformation, and grain growth of two-dimensional binary colloidal crystals: a phase field crystal modeling, Phys. Rev. Mater. 3 (2019).

[18] J.Y. Choi, T. Lee, Y. Cheng, Y. Cohen, Observed crystallization induction time in seeded gypsum crystallization, Ind. Eng. Chem. Res. 58 (2019) 23359-23365.

[19] W. Dai, J. Reimann, D. Hanaor, C. Ferrero, Y. Gan, Modes of wall induced granular crystallisation in vibrational packing, Granul. Matter 21 (2019).

[20] T. Palberg, M.R. Maaroufi, A. Stipp, H.J. Schoepe, Micro-structure evolution of wall based crystals after casting of model suspensions as obtained from Bragg microscopy, J. Chem. Phys. 137 (2012).

[21] H.J. Schoepe, P. Wette, Seed- and wall-induced heterogeneous nucleation in charged colloidal model systems under microgravity, Phys. Rev. E 83 (2011).

[22] G.I. Toth, G. Tegze, T. Pusztai, L. Granasy, Heterogeneous crystal nucleation: the effect of lattice mismatch, Phys. Rev. Lett. 108 (2012).

[23] C. Kohler, R. Backofen, A. Voigt, Relaxation of curvature-induced elastic stress by the Asaro-Tiller-Grinfeld instability, Epl 111 (2015) 6.

[24] C. Kohler, R. Backofen, A. Voigt, Stress induced branching of growing crystals on curved surfaces, Phys. Rev. Lett. 116 (2016) 5.

[25] K. Sandomirski, S. Walta, J. Dubbert, E. Allahyarov, A.B. Schofield, H. Lowen, W. Richtering, S.U. Egelhaaf, Heterogeneous crystallization of hard and soft spheres near flat and curved walls, Eur. Phys. J.-Spec. Top. 223 (2014) 439-454.

[26] L. Ma, X.P. Liu, A.K. Soh, L.H. He, C.Z. Wu, Y. Ni, Growth of curved crystals: competition between topological defect nucleation and boundary branching, Soft Matter 15 (2019) 4391-4400.

[27] N.A. Garcia, R.A. Register, D.A. Vega, L.R. Gomez, Crystallization dynamics on curved surfaces, Phys. Rev. E 88 (2013) 7.

[28] S. Xu, H. Zhou, Z. Sun, J. Xie, Formation of an fcc phase through a bcc metastable state in crystallization of charged colloidal particles, Phys. Rev. E 82 (2010).

[29] H. Zhou, S. Xu, Z. Sun, X. Du, J. Xie, Rapid determination of colloidal crystal's structure by reflection spectrum, Colloids Surf. A-Physicochem. Eng. Asp. 375 (2011) 50-54.

[30] H. Zhou, S. Xu, Z. Sun, X. Du, L. Liu, Kinetics study of crystallization with the disorder-bcc-fcc phase transition of charged colloidal dispersions, Langmuir 27 (2011) 7439-7445.

[31] H. Zhou, S. Xu, Z. Sun, Real-time in situ observation of shear modulus evolution during Ostwald ripening of colloidal crystallization, J. Cryst. Growth 502 (2018) 35-40.

[32] A. Engelbrecht, R. Meneses, H.J. Schoepe, Heterogeneous and homogeneous crystal nucleation in a colloidal model system of charged spheres at low metastabilities, Soft Matter 7 (2011) 5685-5690.

[33] E. Allahyarov, K. Sandomirski, S.U. Egelhaaf, H. Loewen, Crystallization seeds favour crystallization only during initial growth, Nat. Commun. 6 (2015)

[34] A. Esztermann, M. Heni, H. Loewen, J. Klier, M. Sohaili, P. Leiderer, Triple-point wetting on rough substrates, Phys. Rev. Lett. 88 (2002) 055702. 